\title{
Percepción de prácticas parentales y estrategias de aprendizaje en estudiantes secundarios
}

\author{
Perception of Parental Practices and Learning Strategies in \\ High School Students
}

\author{
Nancy Marlene Malander \\ Instituto Superior Adventista de Misiones, Misiones, Argentina
}

\begin{abstract}
Resumen: La presente investigación tuvo por objetivo conocer, mediante un diseño correlacional, el valor predictivo de las prácticas parentales percibidas por los adolescentes, sobre sus hábitos de estudio y estrategias de aprendizaje. Participaron 234 adolescentes argentinos, de ambos sexos, que cursaban sus estudios desde primero a quinto año del nivel medio. Para obtener los datos, se utilizó la Versión Abreviada del Inventario de la Percepción de los Hijos Acerca de las Relaciones con sus Padres para Adolescentes (Richaud de Minzi, 2005), la cual es una adaptación argentina del Children's Report of Parental Behavior Inventory (Schaefer, 1965); y el Inventario de Estrategias de Aprendizaje y Estudio (Strucchi, 1991), adaptación argentina del Learning and Study Strategies Inventory (Weinstein \& Palmer, 1988). Los resultados revelaron que los alumnos que perciben un alto control patológico o una autonomía extrema otorgada por los padres, presentan un menor desarrollo de estrategias de aprendizaje; mientras que los alumnos que los perciben como aceptantes, manifiestan un mejor desarrollo y utilización de las mismas.
\end{abstract}

Palabras clave: prácticas parentales, estrategias de aprendizaje, adolescentes.
Abstract: The aim of this research study was to know, through a correlational design, the predictive value of parental practices perceived by adolescents regarding their study habits and learning strategies. A total of 234 male and female Argentinian adolescents, from 8th through 12th grade participated in the study. In order to obtain the data, an abbreviated Argentinian version (Versión Abreviada del Inventario de la Percepción de los Hijos Acerca de las Relaciones con sus Padres para Adolescentes; Richaud de Minzi, 2005) of Schaefer's Children's Report of Parental Behavior Inventory (1965) for adolescents was used, as well as an Argentinian version (Inventario de Estrategias de Aprendizaje y Estudio; Strucchi, 1991) of the Learning and Study Strategies Inventory, developed by Weinstein and Palmer (1988). Results revealed that students who perceive a high level of pathological control or are granted extreme autonomy by their parents, show less development of learning strategies. Whereas students who perceive their parents as accepting, show a better development and usage of learning strategies.

Keywords: parental practices, learning strategies, adolescents.

Contacto: N. M. Malander. Instituto Superior Adventista de Misiones, Av. Rusia 410, Leandro N. Alem, CP 3315, Misiones, Argentina. Correo electrónico: marlenemalander@gmail.com

Cómo citar: Malander, N. M. (2016). Percepción de prácticas parentales y estrategias de aprendizaje en estudiantes secundarios. Revista de Psicología, 25(1), 1-19.

http://dx.doi.org/10.5354/0719-0581.2016.42098 


\section{Introducción}

En las últimas décadas se ha desarrollado una gran cantidad de investigaciones que ponen de manifiesto la relación entre los estilos o prácticas parentales y el rendimiento académico de los hijos (Cerezo, Casanova, de la Torre, \& Carpio, 2011; Dornbusch, Ritter, Leiderman, Roberts, \& Fraleigh, 1987; Glasgow, Dornbush, Troyer, Steinberg, \& Ritter, 1997; Nyarko, 2011; Pelegrina, García Linares, \& Casanova, 2002; Peralbo Uzquiano \& Fernández Amado, 2003; Steinberg, Lamborn, Darling, Mounts, \& Dornbusch, 1994; Steinberg, Lamborn, Dornbusch, \& Darling, 1992; Vallejo Casarín, \& Mazadiego Infante, 2006). Dichas investigaciones han reconocido la importancia del entorno familiar como uno de los factores que incide en el desarrollo del individuo, a lo largo de todas las edades.

Durante la adolescencia, se producen ciertos cambios en la posición relativa de la persona frente a la familia, los iguales y la sociedad. A pesar de estos cambios, los padres siguen desempeñando un papel muy importante en el proceso de socialización de sus hijos (Grotevant, 1998); y su influencia se extiende a distintos ámbitos, entre ellos el escolar. De hecho, si las condiciones familiares no son propicias, pueden derivarse consecuencias desfavorables para el hijo, tales como una disminución de la motivación y del rendimiento académico, que finalmente conducirán al fracaso escolar (Eccles, Lord, \& Midgley, 1991).

\section{Estilos y dimensiones de crianza parental}

Según Coloma (1993) los estilos parentales son esquemas prácticos que agrupan las numerosas pautas educativas de crianza en unas pocas dimensiones básicas; estas pautas, al combinarse entre sí, dan lugar a tipos habituales de educación familiar.
Fue Baumrind $(1971,1991)$ quien propuso una de las tipologías de estilos de crianza más aceptada en la actualidad: el autoritativo o democrático, el autoritario y el permisivo; a los que, posteriormente, Maccoby y Martin (1983) agregaron un cuarto estilo, el denominado como no implicado, indiferente o negligente. Estos estilos parentales se centran en dos elementos principales: el nivel de exigencia o control, y el afecto, respuesta o nivel de responsabilidad de los padres.

En sus investigaciones, la paternidad autoritativa o democrática es un estilo parental flexible (estos conceptos abarcan padres y madres), en el que se permite a los hijos un elevado grado de libertad. Comprende prácticas de apoyo emocional, concesión de autonomía, comunicación bidireccional; combinando la firmeza con apoyo y protección (Baumrind, 1971, 1991). Los padres democráticos son exigentes y manifiestan un alto control, pero atienden las necesidades de sus hijos (alta respuesta). Son asertivos, pero no intrusivos ni restrictivos.

Los adolescentes que perciben a sus padres como autoritativos o democráticos presentan un mejor ajuste psicológico y una mejor respuesta emocional, en relación con los que los perciben como autoritarios o permisivos (Osorio de Rebellón Yhon, Rivas Borrell, de Irala Estévez, Calatrava, \& López del Burgo, 2009).

El estilo autoritario comprende a padres exigentes (alto control), pero que no se preocupan por las necesidades de los hijos (baja respuesta). Las normas que imponen no pueden ser negociadas, limitando el grado de autonomía de sus hijos. Las órdenes deben ser obedecidas sin explicación. Estos padres presentan un alto grado de control, pero baja aceptación (Baumrind, 1991). 
El estilo permisivo (bajo control, alta respuesta), en tanto, lo constituyen los padres que son poco exigentes, pero responden a las necesidades de los hijos. Establecen pocas reglas y consultan con ellos a la hora de tomar decisiones, siendo muy afectuosos y cálidos en el trato. Son tolerantes, pero no ejercen suficiente control o autoridad, permitiendo un grado máximo de autonomía (Baumrind, 1971). Permiten la autorregulación de sus hijos y evitan la confrontación (Baumrind, 1991).

Por otra parte, el estilo negligente, postulado por Maccoby y Martin (1983), comprende a los padres que ejercen bajo control y baja respuesta a las necesidades de los hijos. No se involucran afectivamente y no se preocupan por establecer normas, pues estas implicarían diálogo y control.

El estilo parental, sin embargo, es un concepto multidimensional, por lo que, además de las dimensiones de afecto y control (Baumrind, 1991; Maccoby \& Martin, 1983), otros autores han estudiado variables como la autonomía (Lewis, 1981), el control psicológico (Oliva, Parra, \& Arranz, 2008) y la comunicación parento-filial (Kerr \& Stattin, 2000), entre otras; las que deberían ser tenidas en cuenta al momento de analizar la influencia de la educación parental sobre el desarrollo y desempeño de los hijos.

Darling y Steinberg (1993) sugieren que el estudio de las dimensiones de calidez, exigencia paterna y autonomía, por separado, resultan mejores indicadores de las características de crianza que las tipologías de estilos parentales; siendo los resultados de las relaciones entre variables más fáciles de interpretar que al utilizar una tipología, en la que no se puede identificar exactamente el componente de la combinación responsable de los resultados.
Por su parte, según Stewart y Bond (2002), las dimensiones parentales son universales, siendo centrales en este trabajo: a) la aceptación, b) el control y c) el respeto por la individualidad o autonomía; cada una de las cuales incluye, a su vez, formas más específicas de interacción (Richaud de Minzi, 2002). Dichas dimensiones corresponden a receptividad parental (parental responsiveness) y exigencia parental (parental demandingness), de Baumrind (1967), y no comprometido o negligente (uninvolved or neglectful parenting), de Maccoby y Martin (1983).

Los padres que se ubican dentro de la dimensión de aceptación, son aquellos que mantienen una actitud cálida y sensible hacia las necesidades de los hijos; son percibidos con alto compromiso, afectuosos y protectores, que promueven una comunicación bidireccional, manteniéndose próximos en momentos de crisis, tratándose de una relación positiva ( $\mathrm{Ri}$ chaud de Minzi, 2002).

Por su parte, el control implica el cumplimiento de las demandas y la vigilancia de las conductas de los hijos. Sin embargo, cuando este se vuelve intrusivo y manipulador, con el desarrollo de sentimientos de culpa y retiro de afecto, se habla de control hostil o patológico, que refleja una relación negativa (Richaud de Minzi, 2002, 2005).

Por último, la autonomía implica el respeto a la individualidad de los adolescentes, sin embargo, cuando es excesiva guarda relación con experiencias familiares de rechazo o frialdad, desvinculación afectiva y percepciones de menor cohesión y flexibilidad familiar (Oliva \& Parra, 2001). Richaud de Minzi (2005) la denomina autonomía extrema, constituyéndose en una percepción negativa de la relación con los padres, que incluye la disciplina laxa. 
Muchos autores consideran la relación entre estilos parentales y rendimiento académico, sin embargo, dicha relación es indirecta; ya que los estilos parentales inciden sobre las características cognitivas y motivacionales implicadas en el aprendizaje, relacionándose estas $-\mathrm{a}$ su vez- con el rendimiento académico posterior (Pérez Sánchez \& Castejón Costa, 1998). Se ha relacionado al estilo democrático con el desarrollo de la motivación intrínseca, y a los estilos autoritario y permisivo, con una orientación motivacional extrínseca (González, Holbein, \& Quilter, 2002).

Pelegrina et al. (2002) agregan, además, que los hijos que perciben a sus padres como democráticos o permisivos se consideran más competentes que aquellos que los perciben como autoritarios o negligentes. Por tal motivo, resulta interesante indagar de qué manera se relacionan las distintas dimensiones parentales con ciertas estrategias y hábitos de estudio de los hijos.

\section{Estrategias de aprendizaje y hábitos de estudio}

Para que los aprendizajes sean significativos y trascendentes, se requiere que el alumno adquiera procedimientos que le permitan asimilar y estructurar el propio proceso de aprender, y el autocontrol de las herramientas y hábitos que favorezcan el éxito académico (Márquez, 1995).

Aprender a estudiar y a utilizar técnicas de estudio es algo fundamental, por lo que conocer los hábitos de estudio y estrategias de aprendizaje de los alumnos es algo importante y trascendente (Sobrado Fernández, Cauce Santalla, \& Rial Sánchez, 2002).
Las estrategias de aprendizaje brindan al alumno herramientas para controlar y regular sus procesos mentales en este ámbito, lo que le permite mejorar su rendimiento y su capacidad de aprender autónomamente a partir de sus propios recursos (Monereo \& Castelló, 1997).

El término estrategia procede del ámbito militar y refiere al arte de dirigir grandes movimientos militares; donde el estratega proyectaba, ordenaba y dirigía las operaciones militares para conseguir la victoria. Cada uno de los pasos que forman una estrategia, son llamados técnicas o tácticas, las que pueden ser utilizadas de forma más o menos mecánica. Sin embargo, las estrategias son siempre conscientes e intencionales, dirigidas a un objetivo relacionado con el aprendizaje (Monereo, Castelló, Clariana, Palma, \& Pérez, 1999).

Según Nisbet y Shucksmith (1987), la estrategia es una guía de las acciones a seguir y es anterior a la elección de cualquier procedimiento para actuar. De modo similar, Weinstein y Mayer (1986) la definen como aquellas conductas y pensamientos que un alumno utiliza durante el aprendizaje, con la intención de influir en su proceso de codificación. Para Beltrán (1993), la estrategia representa a aquellas actividades $\mathrm{u}$ operaciones mentales empleadas para facilitar la adquisición de conocimiento.

La toma consciente de decisiones facilita el aprendizaje significativo, permitiendo que los alumnos establezcan relaciones significativas entre lo que ya saben y la nueva información (Ausubel, 1963). El alumno aprende no solo cómo utilizar determinados procedimientos, sino cuándo y por qué debe utilizarlos (Monereo \& Castelló, 1997). 
Uno de los componentes del aprendizaje autorregulado, son las estrategias cognitivas y metacognitivas (Pintrich, 1999). Las estrategias cognitivas son aquellas que le permiten al alumno la realización de las tareas académicas para lograr el aprendizaje; en tanto que las metacognitivas, permiten que el alumno conozca y controle los procesos psicológicos que le ayudan a aprender.

Considerando los cambios en las políticas educativas, introducidos por la nueva Ley de educación en Argentina $\mathrm{N}^{\circ} 26.206$ que establece que la educación secundaria debe analizar y desarrollar las capacidades de estudio, aprendizaje e investigación de los alumnos, como condición para acceder al aprendizaje a lo largo de toda la vida; es decir, promover la adquisición de herramientas para aprender a aprender, que implica el desarrollo de estrategias de aprendizaje autorregulado-; y conociendo los múltiples estudios acerca de la influencia de la familia en el aprendizaje (sobre todo la percepción que tienen los hijos de la relación con sus padres, y no el comportamiento real de los mismos), la presente investigación se planteó el objetivo de analizar si la percepción de las diferentes dimensiones parentales, desde la propia valoración del hijo, predice significativamente las estrategias y hábitos de estudio en los adolescentes del nivel secundario.

\section{Metodología}

\section{Participantes}

Se llevó a cabo un estudio correlacional del que participaron, incidental y voluntariamente, 234 estudiantes (99 varones y 135 mujeres) del nivel secundario de un instituto de gestión privada de la provincia de Misiones, ubicada en la región noreste de la República Argentina. Del total de encuestados, 48 alumnos $(20,5 \%)$ cursaban el primer año, 39 alumnos $(16,7 \%)$ el segundo año, 58 alumnos $(24,8 \%)$ el tercer año, 45 alumnos $(19,2 \%)$ al cuarto año y 44 alumnos $(18,8)$ el quinto y último curso de educación secundaria. Las edades de los sujetos oscilaron entre los 13 y 21 años, siendo la moda los 16 años con 48 sujetos $(20,5 \%)$; seguida de los 15 años, con 47 sujetos $(20,1 \%)$. La media general fue de 15,64 años $(D E=1,7)$.

\section{Instrumentos}

Se utilizó la versión abreviada del Children's Report of Parental Behavior Inventory de Schaefer (1965), adaptado a Argentina por Richaud de Minzi (2005; Inventario de la Percepción de los Hijos Acerca de las Relaciones con sus Padres para Adolescentes). Dicho inventario evalúa las siguientes dimensiones parentales desde la percepción de los hijos: a) aceptación, b) control patológico y c) autonomía extrema. Dicho cuestionario consta de 32 ítems, en versiones separadas para la percepción de las prácticas maternas y la percepción de las prácticas paternas. Las opciones de respuesta a cada una de las afirmaciones son: sí, más $o$ menos y no. El coeficiente alfa de Cronbach, para cada uno de los tres factores respecto de la madre, arrojó índices de fiabilidad de ,81 en aceptación; ,83 en control patológico y ,75 en autonomía extrema; y de ,82 en aceptación; ,84 en control patológico y ,73 en autonomía extrema, respecto del padre (Richaud de Minzi, 2005).

Se empleó, además, el Inventario de Estrategias de Aprendizaje y Estudio una Adaptación Argentina (Strucchi, 1991), adaptación del Learning and Study Strategies Inventory, elaborado por Weinstein y Palmer (1988), que presenta un índice de 
fiabilidad alfa de Cronbach de ,88 para el instrumento total (Castañeiras, Guzmán, Posada, Ricchini, \& Strucchi, 1999). Dicho cuestionario consta de 77 ítems, con opciones de respuesta codificadas con una escala de tipo Likert que va de 0 a 4 puntos. Las respuestas de cada ítem se suman agrupándose en 10 escalas:

1) Actitud, que evalúa las actitudes generales de los estudiantes y su motivación para tener éxito en el estudio; principalmente, en situaciones de aprendizaje autónomo.

2) Motivación, que mide el grado de responsabilidad para llevar a cabo tareas específicas relacionadas con el aprendizaje.

3) Administración del tiempo, que mide la utilización y organización del tiempo destinado a tareas académicas.

4) Ansiedad, que evalúa el grado de preocupación de los estudiantes por su desempeño académico.

5) Concentración, que mide la capacidad que tienen los estudiantes para dirigir su atención hacia las tareas escolares.

6) Procesamiento de la información, que evalúa si los estudiantes pueden crear elaboraciones verbales y organizaciones para fomentar el entendimiento.

7) Selección de ideas principales, que enfatiza la habilidad para escoger información clave.

8) Técnicas de ayuda al estudio, que mide la habilidad para usar o crear técnicas que permitan comprender, estudiar y recordar información.

9) Autoevaluación, que se centra en actividades de preparación y revisión de clases y pruebas.

10) Estrategias de prueba, que incluyen el conocimiento acerca de las características del examen y cómo preparar un efectivo plan para rendirlo.

\section{Procedimientos}

Una vez obtenido el consentimiento informado de los directivos del establecimiento educativo en el que se llevó a cabo la investigación, y de los padres o tutores legales de los adolescentes, se solicitó a estos últimos su participación voluntaria. Previamente a la entrega de los cuestionarios, se realizó una detallada explicación de la investigación y sus objetivos, así como también de los procedimientos a seguir para contestar los test.

Se explicó claramente que la participación sería anónima, haciendo hincapié en la sinceridad de las respuestas y la realización a conciencia de las pruebas. En el caso del cuestionario de percepción de las relaciones con los padres, se explicó que aquellos que tuvieran solo un progenitor debían completar uno de los cuestionarios; en el caso de que alguien contara con alguna persona que consideraba que cumplía con el rol del progenitor ausente, se les dijo que podrían completar ambos cuestionarios.

Las respuestas recogidas mediante la adaptación del Learning and Study Strategies Inventory y la adaptación del Children's Report of Parental Behavior Inventory, fueron ingresadas al programa Statistical Package for the Social Sciences (SPSS, versión 22), para el correspondiente análisis estadístico. En primer lugar, se sumaron las respuestas dadas a cada ítem para obtener los totales brutos de cada escala de estrategias de aprendizaje y de cada dimensión parental. Luego, se aplicaron análisis de regresión lineal múltiple para conocer qué dimensiones parentales (paternas y maternas) predecían el desarrollo de las distintas estrategias de aprendizaje. Se fijó un nivel de significación del $5 \%$. 


\section{Resultados}

A continuación, se exponen los resultados obtenidos del análisis de regresión múltiple para evaluar el poder predictivo de las percepciones de los alumnos acerca de las relaciones con sus padres (variable predictora) y las estrategias de aprendizaje que poseen (variable dependiente).

Percepción de la relación con los padres $y$ actitud de los hijos hacia el estudio

Mediante el análisis de regresión múltiple, se encontraron dos variables paternas que predijeron estadísticamente la actitud de los hijos hacia el estudio, $R^{2}=105 ; F(3$, $225)=8,801 ; p<, 05$. A menor control patológico $(\beta=-, 287 ; p<, 05)$ y autonomía extrema otorgada por el padre $(\beta=$ $, 141 ; p<, 05)$, le corresponde mayor puntaje en la escala de actitud hacia el estudio.

El análisis de las dimensiones maternas mostró resultados similares, aunque con un coeficiente de determinación bajo, $R^{2}=$ ,056, $F(3,230)=4,542, p<, 05$. A menor control patológico $(\beta=-, 212 ; p<, 05)$ y autonomía extrema materna $(\beta=-, 141 ; p<$ ,05), le corresponde también un mayor puntaje en la escala de actitud hacia el estudio. Es decir, los padres que ejercen un menor control patológico y una menor autonomía extrema, logran generar y desarrollar en sus hijos actitudes positivas y de mayor interés hacia el estudio (ver tabla 1).

Percepción de la relación con los padres y motivación de los hijos hacia el estudio

Al realizar el análisis estadístico, se encontraron tres variables paternas que explicaron la motivación de los hijos hacia el estudio: $R^{2}=, 101 ; F(3,225)=8,466 ; p<$ ,05. La mayor aceptación paterna aumenta el puntaje obtenido en la escala de motivación $(\beta=, 140 ; p<, 05)$, mientras que el mayor control patológico disminuye dichos puntajes $(\beta=-, 164 ; p<, 05)$. En el caso de la autonomía extrema o negligencia paterna la relación también es inversa $(\beta=-, 242 ; p<, 05)$. Los alumnos que perciben a su padre como controlador o negligente en cuanto a la disciplina y relación con ellos, desarrollan menor motivación para el cumplimiento de las tareas escolares, en cambio, los alumnos que perciben aceptación y afecto positivo se muestran más motivados hacia el estudio.

Tabla 1

Regresión múltiple para las dimensiones de los estilos parentales y actitud hacia el estudio

\begin{tabular}{lccc}
\hline Estilos parentales & $\mathrm{B}$ & $\beta$ & $I C 95 \%$ \\
\hline Padres & & & \\
Aceptación &, 110 &, 086 & {$[-, 052 ;, 271]$} \\
Control patológico &,- 251 &,$- 287^{*}$ & {$[-, 362 ;-, 139]$} \\
Autonomía extrema &,- 227 &,$- 141^{*}$ & {$[-, 431 ;-, 023]$} \\
Madres & & & \\
Aceptación &,- 015 &,- 009 & {$[-, 242 ;, 213]$} \\
Control patológico &,- 182 &,$- 212^{*}$ & {$[-, 298 ;-, 067]$} \\
Autonomía extrema &,- 237 &,$- 141^{*}$ & {$[-, 451 ;-, 024]$} \\
\hline
\end{tabular}

Nota: $\mathrm{B}=$ coeficiente de regresión parcial; $\beta=$ coeficiente de regresión parcial estandarizado; $I C=$ Intervalo de Confianza.

${ }^{*} p<, 05$ 
En el caso de la percepción del estilo materno, los resultados no mostraron valor predictivo sobre la escala de motivación (ver tabla 2).

Percepción de la relación con los padres y administración del tiempo por parte de los hijos

Los resultados mostraron que existen tres variables paternas que predijeron significativamente la adecuada administración del tiempo dedicado al estudio, $R^{2}=, 109 ; F(3,225)=9,196 ; p<, 05 . \mathrm{Se}$ observó que, a mayor aceptación paterna, mejor es la administración del tiempo $(\beta=, 180 ; p<, 05)$; mientras que a mayor control patológico $(\beta=-, 150 ; p<$ ,05) y autonomía extrema $(\beta=-, 233 ; p<$ ,05), menos adecuada es la administración del tiempo.

Los alumnos que perciben aceptación por parte del padre, menor control patológico y menor autonomía extrema; presentan un mejor uso de su tiempo, planificando sus actividades para el logro de mejores resultados académicos (ver tabla 3 ).

Tabla 2

Regresión múltiple para las dimensiones de los estilos parentales y motivación hacia el estudio

\begin{tabular}{lrcc}
\hline Estilos parentales & $\mathrm{B}$ & $\beta$ & $I C 95 \%$ \\
\hline Padres & & & \\
$\quad$ Aceptación &, 187 &, $140^{*}$ & {$[, 018 ;, 355]$} \\
Control patológico &,- 149 &,$- 164^{*}$ & {$[-, 266 ;-, 033]$} \\
Autonomía extrema &,- 406 &,$- 242^{*}$ & {$[-, 620 ;-, 193]$} \\
Madres & & & \\
Aceptación &, 157 &, 088 & {$[-, 083 ;, 397]$} \\
Control patológico &,- 083 &,- 093 & {$[-, 205 ;, 039]$} \\
Autonomía extrema &,- 195 &,- 112 & {$[-, 421 ;, 031]$} \\
\hline
\end{tabular}

Nota: $\mathrm{B}=$ coeficiente de regresión parcial; $\beta=$ coeficiente de regresión parcial estandarizado; $I C=$ Intervalo de Confianza.

${ }^{*} p<, 05$

Tabla 3

Regresión múltiple para las dimensiones de los estilos parentales y administración del tiempo

\begin{tabular}{lccc}
\hline Estilos parentales & $\mathrm{B}$ & $\beta$ & $I C 95 \%$ \\
\hline Padres & & & \\
$\quad$ Aceptación &, 217 &, $180^{*}$ & {$[, 065 ;, 369]$} \\
Control patológico &,- 123 &,$- 150^{*}$ & {$[-, 228 ;-, 018]$} \\
$\quad$ Autonomía extrema &,- 355 &,$- 233^{*}$ & {$[-, 547 ;-, 163]$} \\
Madres & & & \\
Aceptación &,- 084 &,- 052 & {$[-, 304 ;, 137]$} \\
Control patológico &,- 087 &,- 107 & {$[-, 199 ;, 025]$} \\
Autonomía extrema &,- 144 &,- 090 & {$[-, 351 ;, 063]$} \\
\hline
\end{tabular}

Nota: $\mathrm{B}=$ coeficiente de regresión parcial; $\beta=$ coeficiente de regresión parcial estandarizado $; C=$ Intervalo de Confianza.

${ }^{*} p<, 05$ 
Para el caso de la percepción de los estilos maternos, y de la administración del tiempo, no se encontraron variables predictoras (tal como se puede apreciar en la tabla 3).

Percepción de la relación con los padres y manejo de la ansiedad o preocupación de los hijos por el rendimiento escolar

Los resultados indicaron una sola variable paterna que predijo estadísticamente la ansiedad, aunque con un porcentaje de variancia explicada muy bajo: $R^{2}=, 039$; $F(3,225)=3,008 ; p<, 05$. Se observó que, a mayor control patológico del padre, menor es el puntaje obtenido en manejo de la ansiedad $(\beta=-, 161 ; p<$ ,05).

Cabe recordar que los puntajes bajos obtenidos en manejo de ansiedad son indicadores de individuos que, posiblemente, se hallan preocupados por no desempeñarse correctamente, o por pensar que no son capaces de lograr un buen desempeño.
Mientras que los puntajes altos obtenidos en esta escala, demuestran un manejo productivo de la ansiedad, es decir, son capaces de utilizarla como motor que los moviliza a concretar la meta, focalizándose en la tarea.

Para la percepción de los estilos maternos, los resultados también mostraron una única variable significativa, aunque nuevamente con un bajo poder predictivo, $R^{2}=, 048 ; F(3,230)=3,885 ; p<, 05$. A mayor control patológico de la madre, menor es el puntaje obtenido en manejo de la ansiedad que presenta el alumno ante las exigencias escolares $(\beta=-, 172$; $p<, 05)$.

El control patológico o excesivo, tanto del padre como de la madre, contribuyó a explicar -aunque escasamente- los sentimientos de ansiedad de los hijos ante los exámenes y otros requerimientos escolares; los que pueden dificultar la realización de los mismos, generando pensamientos de falta de capacidad para el logro de sus metas (ver tabla 4).

Tabla 4

Regresión múltiple para las dimensiones de los estilos parentales y ansiedad

\begin{tabular}{lccc}
\hline Estilos parentales & $\mathrm{B}$ & $\beta$ & $I C 95 \%$ \\
\hline Padres & & & \\
Aceptación &,- 119 &,- 082 & {$[-, 311 ;, 072]$} \\
Control patológico &,- 161 &,$- 161^{*}$ & {$[-, 293 ;-, 028]$} \\
Autonomía extrema &, 139 &, 075 & {$[-, 103 ;, 380]$} \\
Madres & & & \\
Aceptación &,- 321 &,- 166 & {$[-, 580 ;-, 062]$} \\
Control patológico &,- 168 &,$- 172^{*}$ & {$[-, 300 ;-, 037]$} \\
Autonomía extrema &, 162 &, 085 & {$[-, 082 ;, 405]$} \\
\hline
\end{tabular}

Nota: $\mathrm{B}=$ coeficiente de regresión parcial; $\beta=$ coeficiente de regresión parcial estandarizado; $I C=$ Intervalo de Confianza.

${ }^{*} p<, 05$. 
Percepción de la relación con los padres y concentración de los hijos a la hora de estudiar

Los resultados mostraron tres dimensiones paternas que predijeron significativamente la concentración, $R^{2}=, 118$; $F(3,225)=9,990 ; p<, 05$. Ante una mayor aceptación por parte del padre, se observó una mayor concentración a la hora de estudiar $(\beta=, 139 ; p<, 05)$. En cambio, a mayor control patológico $(\beta=$ -,216; $p<, 05)$ y autonomía extrema paterna $(\beta=-, 238 ; p<, 05)$, menor es la capacidad de concentración en el estudio (ver tabla 5).

En el caso de la madre, se encontró una variable que predijo leve, pero significativamente, la concentración: $R^{2}=, 044 ; F(3$, $225)=3,553 ; p<, 05$. A mayor control patológico, menor es la concentración y focalización que logra el hijo al estudiar $(\beta=-, 216 ; p<, 05)$.
Percepción de la relación con los padres y procesamiento de la información escolar de los hijos

Sobre la base del análisis de regresión múltiple, no se encontraron variables paternas predictoras de la escala de procesamiento de la información (como se puede apreciar en la tabla 6).

En el caso de la madre, se encontró una dimensión que predijo significativamente esta estrategia cognitiva, aunque con un coeficiente de determinación bajo: $R^{2}=$ , $047 ; F(3,230)=3,800 ; p<, 05$.

A mayor aceptación por parte de la madre, mayor es el desarrollo de la estrategia de procesamiento de la información $(\beta=$ ,224; $p<, 05)$; es decir, las madres aceptantes promueven en sus hijos la capacidad de procesar adecuadamente la información, la adquisición del conocimiento y el razonamiento.

Tabla 5

Regresión múltiple para las dimensiones de los estilos parentales y concentración

\begin{tabular}{lrcc}
\hline Estilos parentales & $\mathrm{B}$ & $\beta$ & $I C 95 \%$ \\
\hline Padres & & & \\
Aceptación &, 192 &, $139^{*}$ & {$[, 018 ;, 365]$} \\
Control patológico &,- 203 &,$- 216^{*}$ & {$[-, 323 ;-, 083]$} \\
Autonomía extrema &,- 415 &,$- 238^{*}$ & {$[-, 634 ;-, 197]$} \\
Madres & & & \\
Aceptación &,- 059 &,- 032 & {$[-, 309 ;, 190]$} \\
Control patológico &,- 202 &,$- 216^{*}$ & {$[-, 329 ;-, 076]$} \\
Autonomía extrema &,- 130 &,- 071 & {$[-, 364 ;, 105]$} \\
\hline
\end{tabular}

Nota: $\mathrm{B}=$ coeficiente de regresión parcial; $\beta=$ coeficiente de regresión parcial estandarizado; $I C=$ Intervalo de Confianza.

${ }^{*} p<, 05$ 
Tabla 6

Regresión múltiple para las dimensiones de los estilos parentales y procesamiento de la información

\begin{tabular}{|c|c|c|c|}
\hline Estilos parentales & B & $\beta$ & $I C 95 \%$ \\
\hline \multicolumn{4}{|l|}{ Padres } \\
\hline Aceptación & , 166 &, 125 & {$[-, 010 ;, 342]$} \\
\hline Control patológico &, 003 & ,003 & {$[-, 119 ;, 125]$} \\
\hline Autonomía extrema &,- 053 &,- 032 & {$[-, 275 ;, 170]$} \\
\hline \multicolumn{4}{|l|}{ Madres } \\
\hline Aceptación & ,396 & $224^{*}$ & {$[, 159 ;, 634]$} \\
\hline Control patológico &, 057 & ,064 & {$[-, 063 ;, 178]$} \\
\hline Autonomía extrema &,- 096 &,- 055 & {$[-, 319 ;, 128]$} \\
\hline
\end{tabular}

Nota: $\mathrm{B}=$ coeficiente de regresión parcial; $\beta=$ coeficiente de regresión parcial estandarizado; $I C=$ Intervalo de Confianza. ${ }^{*} p<, 05$

Percepción de la relación con los padres y selección de ideas principales

En el caso del padre, se encontró una variable predictora, $R^{2}=, 072 ; F(3,225)=$ 5,$831 ; p<, 05$. A mayor control patológico, menor es el desarrollo de la estrategia de selección de ideas principales $(\beta=$ ,240; $p<, 05)$.

En cuanto a la percepción de la relación con la madre, los resultados se mostraron en la misma línea, $R^{2}=, 065 ; F(3,230)=$ 5,$371 ; p<, 05$. A mayor control patológico materno, menor es el desarrollo de la estrategia de selección de ideas principales $(\beta=-, 263 ; p<, 05)$.

En otras palabras, los padres que ejercen menor control patológico promueven en sus hijos la capacidad de reconocer la información significativa al momento de estudiar (ver tabla 7).

Percepción de la relación con los padres y estrategias de ayuda en el estudio

Los resultados mostraron una variable paterna con predicción significativa, $R^{2}=$ , $081 ; F(3,225)=6,566 ; p<, 05$. A mayor aceptación por parte del padre, se presentó un mayor desarrollo de técnicas que ayudan a aprender por parte del hijo $(\beta=$ ,257; $p<, 05)$ (ver tabla 8 ).

En cuanto a la percepción del estilo materno, se encontraron dos variables predictivas, $R^{2}=, 057 ; F(3,230)=4,671 ; p<$ ,05. A mayor aceptación materna, mayor es el puntaje obtenido en la estrategia de técnicas de ayuda en el estudio $(\beta=, 188$; $p<, 05)$, mientras que a mayor autonomía extrema, menor es el desarrollo de la estrategias que ayudan a aprender los diferentes materiales escolares $(\beta=-, 135 ; p<$ ,05) (ver tabla 8).

Percepción de la relación con los padres y estrategias de autoevaluación en los hijos

El análisis de regresión múltiple mostró dos variables paternas significativas, $R^{2}=$ , $071 ; F(3,225)=5,755 ; p<, 05$. A mayor aceptación por parte del padre, mayor desarrollo de la estrategia de autoevaluación ( $\beta$ $=, 170 ; p<, 05) \mathrm{y}$, contrariamente a lo esperado, a mayor control patológico, mayor es el puntaje obtenido en autoevaluación ( $\beta$ $=, 169 ; p<, 05)($ ver tabla 9$)$. 
Tabla 7

Regresión múltiple para las dimensiones de los estilos parentales y selección de ideas principales

\begin{tabular}{|c|c|c|c|}
\hline Estilos parentales & B & $\beta$ & $I C 95 \%$ \\
\hline \multicolumn{4}{|l|}{ Padres } \\
\hline Aceptación & ,069 & ,079 & {$[-, 044 ;, 181]$} \\
\hline Control patológico &,- 143 &,$- 240^{*}$ & {$[-, 221 ;-, 065]$} \\
\hline Autonomía extrema &,- 107 &,- 097 & {$[-, 249 ;, 035]$} \\
\hline \multicolumn{4}{|l|}{ Madres } \\
\hline Aceptación &,- 070 &,- 060 & {$[-, 225 ;, 085]$} \\
\hline Control patológico &,- 155 &,$- 263^{*}$ & {$[-, 234 ;-, 076]$} \\
\hline Autonomía extrema &,- 107 &,- 093 & {$[-, 253 ;, 039]$} \\
\hline
\end{tabular}

Nota: $\mathrm{B}=$ coeficiente de regresión parcial; $\beta=$ coeficiente de regresión parcial estandarizado; $I C=$ Intervalo de Confianza.

${ }^{*} p<, 05$

Tabla 8

Regresión múltiple para las dimensiones de los estilos parentales y ayudas al estudio

\begin{tabular}{lccc}
\hline Estilos parentales & $\mathrm{B}$ & $\beta$ & $I C 95 \%$ \\
\hline Padres & & & \\
Aceptación &, 379 &, $257^{*}$ & {$[, 190 ;, 568]$} \\
Control patológico &,- 012 &,- 012 & {$[-, 142 ;, 119]$} \\
$\quad$ Autonomía extrema &,- 201 &,- 108 & {$[-, 440 ;, 037]$} \\
Madres & & & \\
Aceptación &, 368 &, $188^{*}$ & {$[, 108 ;, 628]$} \\
Control patológico &,- 050 &,- 050 & {$[-, 182 ;, 082]$} \\
Autonomía extrema &,- 260 &,$- 135^{*}$ & {$[-, 504 ;-, 015]$} \\
\hline
\end{tabular}

Nota: $\mathrm{B}=$ coeficiente de regresión parcial; $\beta=$ coeficiente de regresión parcial estandarizado; $I C=$ Intervalo de Confianza.

${ }^{*} p<, 05$

En vista de este resultado contradictorio, se realizó un análisis de regresión múltiple adicional, de estimación por pasos (stepwise), considerando como variables independientes cada uno de los ítems de control patológico paterno. Los resultados mostraron dos ítems que predijeron positiva y significativamente la autoevaluación, $R^{2}=, 081, \mathrm{~F}(2,226)=9,937, \mathrm{p}<$ ,05, "Me controla cuidadosamente los amigos" $(\beta=, 196 ; p<, 05)$ y "Decide con qué amigos puedo relacionarme" $(\beta=$ ,149; $\mathrm{p}<, 05)$, quedando los demás ítems eliminados del modelo.
En cuanto a las dimensiones maternas, se encontró una variable predictora, pero con un porcentaje de variancia muy bajo, $R^{2}=, 039 ; F(3,230)=3,132 ; p<, 05$. A mayor aceptación materna, mayor es el resultado obtenido en la estrategia de autoevaluación $(\beta=, 187 ; p<, 05)$. Los hijos que perciben mayor aceptación materna, están mejor preparados para autorrevisar los contenidos que han aprendido y monitorear la comprensión de los temas de clases (como lo muestra la tabla 9). 
Tabla 9

Regresión múltiple para las dimensiones de los estilos parentales y autoevaluación

\begin{tabular}{|c|c|c|c|}
\hline Estilos parentales & B & $\beta$ & $I C 95 \%$ \\
\hline \multicolumn{4}{|l|}{ Padres } \\
\hline Aceptación & ,230 &, $170^{*}$ & {$[, 056 ;, 403]$} \\
\hline Control patológico &, 155 &, $169^{*}$ & {$[, 035 ;, 275]$} \\
\hline Autonomía extrema &,- 207 &,- 122 & {$[-, 426 ;, 012]$} \\
\hline \multicolumn{4}{|l|}{ Madres } \\
\hline Aceptación & ,338 &, $187^{*}$ & {$[, 095 ;, 582]$} \\
\hline Control patológico &, 123 & ,135 & {$[, 000 ;, 247]$} \\
\hline Autonomía extrema &,- 077 &,- 043 & {$[-, 306 ;, 152]$} \\
\hline
\end{tabular}

Nota: $\mathrm{B}=$ coeficiente de regresión parcial; $\beta=$ coeficiente de regresión parcial estandarizado; $I C=$ Intervalo de Confianza. ${ }^{*} p<, 05$

Percepción de la relación con los padres y estrategias de preparación de exámenes

Se encontró, en el caso del padre, una variable que predijo significativamente las estrategias de preparación de exámenes, $R^{2}=, 077 ; F(3,225)=6,248 ; p<, 05$. A mayor control patológico, menor desarrollo de la estrategia de preparación de exámenes $(\beta=-, 262 ; p<, 05)$.

En el caso de la madre, se encontró la misma variable predictiva, $R^{2}=, 103 ; F(3$, $230)=8,784 ; p<, 05$. A mayor control materno patológico, menor es el desarrollo de la estrategia de preparación de exámenes en los hijos $(\beta=-, 337 ; p<, 05)$ (ver tabla 10).

\section{Discusión y conclusiones}

Si bien los resultados obtenidos arrojaron coeficientes de determinación bajos; en la mayoría de los casos menores al 10\%, los resultados están en línea con la teoría y contribuyen a explicar uno de los factores que podría predecir los hábitos de estudio y las estrategias de aprendizaje utilizadas por los alumnos de nivel secundario.

Tabla 10

Regresión múltiple para las dimensiones de los estilos parentales y estrategias de preparación de exámenes

\begin{tabular}{lccc}
\hline Estilos parentales & $\mathrm{B}$ & $\beta$ & $I C 95 \%$ \\
\hline Padres & & &, 049 \\
$\quad$ Aceptación &,- 223 &,$- 262^{*}$ & {$[-, 111 ;, 209]$} \\
Control patológico &,- 180 &,- 114 & {$[-, 333 ;-, 112]$} \\
$\quad$ Autonomía extrema & & & \\
Madres &, 215 &, 128 & {$[, 003 ;, 433]$} \\
Aceptación &,- 284 &,$- 337^{*}$ & {$[, 395 ;-, 174]$} \\
Control patológico &,- 140 &,- 085 & {$[-, 345 ;, 064]$} \\
Autonomía extrema &
\end{tabular}

Nota: $\mathrm{B}=$ coeficiente de regresión parcial; $\beta=$ coeficiente de regresión parcial estandarizado; $\mathrm{IC}=$ Intervalo de Confianza.

${ }^{*} p<, 05$ 
Sobre la base de los resultados de la presente investigación, se pudo concluir que el bajo control patológico por parte del padre predice las estrategias de: actitud positiva, motivación, administración del tiempo, manejo de la ansiedad, concentración, selección de ideas principales y preparación de exámenes. Es decir, los padres que no utilizan el control hostil, la culpa, el retiro de relación, la posesividad o el rechazo hacia sus hijos dentro de sus prácticas parentales, promueven en ellos una actitud positiva hacia el estudio en general; lo que los lleva también a estar motivados a la hora de estudiar, permitiéndoles utilizar, además, otras estrategias de aprendizaje necesarias para un buen logro académico.

Estos resultados coincidieron exactamente con los obtenidos por Concha Lescano (2008) en un estudio realizado con 145 alumnos argentinos de nivel secundario, procedentes de la región central del país.

Además, y llamativamente, en la presente investigación se observó que un alto control patológico predice la estrategia de autoevaluación, que es la única estrategia metacognitiva estudiada. Específicamente, fueron dos los ítems que componen dicha dimensión parental los que explican esta relación entre las variables: "Me controla cuidadosamente los amigos" y "Decide con qué amigos puedo relacionarme". Como puede verse, no se trata de conductas parentales extremadamente hostiles, posesivas o patológicas, como sí podrían ser: "Siempre me está encontrando faltas", "Cuando lo decepciono no quiere saber nada conmigo", "Quiere que me quede en casa para controlarme", "Debo hacer exactamente lo que me dice".

Lo anterior podría indicar que los alumnos que constituyen la presente muestra, podrían no encontrar como algo negativo el hecho de que los padres elijan los amigos con los que se relacionan, sino percibirlo como una forma de protección que, a su vez, repercutiría positivamente en sus estrategias de estudios.

Otra posible explicación es que dichos ítems podrían estar relacionados con lo que Kerr y Stattin (2000) han aportado acerca de un aspecto de la comunicación parento-filial, que denominan revelación (self-disclosure), que se refiere a la tendencia del adolescente a informar espontáneamente acerca de sus amistades, relaciones de pareja, etcétera. Esta revelación puede ser considerada como una estrategia sutil de control parental, en lugar de un control hostil o patológico (Crouter, Bumpus, Davis, \& McHale, 2005; Oliva, 2006).

Por otra parte, el bajo control patológico de la madre predice las estrategias de: actitud positiva, manejo de la ansiedad, concentración, selección de ideas principales y preparación de exámenes. Estos resultados coincidieron con Concha Lescano (2008), aunque dicha autora también encontró que predice la estrategia de administración del tiempo.

En cuanto al control patológico, ya sea paterno o materno, los resultados del presente estudio están en línea con lo obtenido por Erden y Uredi (2008) y Cerezo et al. (2011), cuyos estudios muestran que los hijos que perciben a sus padres como autoritarios, presentan un deficiente uso de habilidades de aprendizaje autorregulado y mayor ansiedad ante los exámenes. La presente investigación puso de manifiesto que el control patológico, entendido como un control hostil, basado en estrategias psicológicas encubiertas como la culpa y el temor, explica en mayor medida el escaso desarrollo o adquisición de las estrategias de aprendizaje. 
La baja autonomía extrema otorgada por parte del padre, predice las estrategias de: actitud positiva, motivación, administración del tiempo y concentración. Esto significa que los padres que no dan una libertad excesiva a sus hijos, ni los exponen a situaciones o responsabilidades para las cuales aún no están preparados, fomentan en ellos el desarrollo de ciertas estructuras cognitivas y hábitos de estudio que les van a permitir tener mejores oportunidades de éxito escolar.

Estos resultados coincidieron, en parte, con los obtenidos por Concha Lescano (2008), en cuanto a las estrategias de actitud y concentración. Del mismo modo, la baja autonomía extrema por parte de la madre predice las estrategias de: actitud positiva y ayuda al estudio. Los hallazgos de la presente investigación coincidieron con lo obtenido por Concha Lescano (2008), en cuanto a la estrategia de actitud positiva.

La aceptación por parte del padre predice, significativamente, las estrategias de: motivación, administración del tiempo, concentración, ayudas en el estudio y autoevaluación. Dichos resultados difirieron de los obtenidos por Concha Lescano (2008), quien concluyó que la aceptación por parte del padre predice únicamente la preparación de los exámenes. La aceptación materna, en tanto, predice las estrategias de: procesamiento de la información, ayudas en el estudio y autoevaluación; coincidiendo con los resultados obtenidos por Concha Lescano (2008), excepto en cuanto a la estrategia de autoevaluación.

Existen varias investigaciones que han demostrado que los adolescentes con padres democráticos, que ofrecen un alto grado de afecto y aceptación, presentan niveles más altos de autoestima, con mayor interés hacia la escuela y mejor ren- dimiento académico; lo que podría estar relacionado con sus hábitos de estudio y estrategias de aprendizaje (Glasgow et al., 1997; Lamborn, Mounts, Steinberg, \& Dornbush, 1991; Pelegrina et al., 2002).

Los resultados obtenidos también coincidieron con Baumrind (1991), en que altos niveles de aceptación e implicación fomentan la autorregulación de los hijos, y que la relación entre actitudes de apoyo de los padres y las conductas de autorregulación de los hijos y el uso de estrategias de aprendizaje, es positiva (Cerezo et al., 2011; Grolnick \& Ryan, 1989).

En consonancia con los resultados obtenidos por Erden y Uredi (2008), se encontró que los padres autoritarios (que ejercen demasiado control sobre sus hijos) o los negligentes (que aplican un sistema de disciplina laxa y exceso de autonomía), fomentan en sus hijos la falta de confianza y un deficiente uso de estrategias de aprendizaje. Estos autores encontraron que los padres autoritativos o democráticos, y los padres permisivos (que coinciden en un alto grado de afecto, que podría asimilarse a la aceptación), son los que facilitan el desarrollo de estrategias cognitivas y metacognitivas; mientras que los padres autoritarios $\mathrm{y}$ negligentes dificultan el desarrollo de dichas estrategias, lo cual podría asimilarse a los padres que ejercen un control patológico o los que otorgan una autonomía extrema.

Los resultados coincidieron con dichos autores, donde los padres autoritativos (que aplican un alto grado de aceptación y estimulan la expresión de la individualidad de sus hijos) logran mayores niveles de concentración en el trabajo.

La aceptación parental -como mostraron los resultados de este trabajo-, sobre todo 
cuando es proporcionada por el padre, no emergió como la principal variable predictora; ya que pareciera ser que las prácticas parentales negativas, de control patológico y autonomía extrema, resultaron ser los predictores más poderosos de las estrategias de aprendizaje de los adolescentes. Esto permite concluir que, para mejorar las estrategias de aprendizaje, no solo se de- ben potenciar las prácticas parentales saludables, sino también se debe tratar de erradicar las prácticas educativas insanas.

El aprendizaje es un proceso complejo, por lo que se recomienda que en futuras investigaciones se aborde el estudio de la relación entre las prácticas parentales y otras variables del aprendizaje escolar.

\section{Referencias}

Ausubel, D. P. (1963). The psychology of meaningful verbal learning. New York, New York: Grune \& Stratton.

Baumrind, D. (1967). Child care practices anteceding three patterns of preschool behavior. Genetic Psychology Monographs, 75(1), 43-88.

Baumrind, D. (1971). Current patterns of parental authority. Developmental Psychology, 4(1), 1-103. http://dx.doi.org/10.1037/h0030372

Baumrind, D. (1991). The influence of parenting style on adolescent competence and substance use. The Journal of Early Adolescence, 11(1), 56-95. http://dx.doi.org/10.1177/0272431691111004

Beltrán, J. (1993). Procesos, estrategias y técnicas de aprendizaje. Madrid, España: Síntesis.

Castañeiras, C., Guzmán, G., Posada, M., Ricchini, M., \& Strucchi, E. (1999). Sobre estrategias de aprendizaje y hábitos de estudio. Revista Iberoamericana de Diagnóstico y Evaluación Psicológica, 8(2), 37-50. Recuperado de http://www.aidep.org/03_ridep/R08/R082.pdf

Cerezo, M. T., Casanova, P. F., de la Torre, M. J., \& Carpio, M. V. (2011). Estilos educativos paternos y estrategias de aprendizaje en alumnos de educación secundaria. European Journal of Education and Psychology, 4(1), 51-61. http://dx.doi.org/10.1989/ejep.v4i1.76

Coloma Medina, J. (1993). Estilos educativos paternos. En J. M. Quintana Cabanas (Coord.), Pedagogía familiar (pp. 45-58). Madrid, España: Narcea.

Concha Lescano, M. (2008). Influencia de los estilos parentales y las creencias de autoeficacia, en las metas académicas y las estrategias de aprendizaje en adolescentes (Tesis inédita de licenciatura). Universidad Adventista del Plata, Entre Ríos, Argentina.

Crouter, A. C., Bumpus, M. F., Davis, K. D., \& McHale, S. M. (2005). How do parents learn about adolescents' experiences? Implications for parental knowledge and adolescent risky behavior. Child Development, 76(4), 869-882. http://dx.doi.org/10.1111/j.1467-8624.2005.00883.x 
Darling, N. \& Steinberg, L. (1993). Parenting style as context: An integrative model. Psychological Bulletin, 113(3), 487-496.

http://psycnet.apa.org/doi/10.1037/0033-2909.113.3.487

Dornbusch, S. M., Ritter, P. L., Leidermann, P. H., Roberts, D. F., \& Fraleigh, M. J. (1987). The relation of parenting style to adolescent school performance. Child Development, 58(5), 1244-1257.

http://dx.doi.org/10.2307/1130618

Eccles, J. S., Lord, S., \& Midgley, C. (1991). What are we doing to early adolescents? The impact of educational contexts on early adolescents. American Journal of Education, 99(4), 521-542.

http://dx.doi.org/10.1086/443996

Erden, M. \& Uredi, I. (2008). The effect of perceived parenting styles on self-regulated learning strategies and motivational beliefs. International Journal about Parents in Education, 2(1), 25-34.

Glasgow, K. L. Dornbusch, S. M. Troyer, L. Steinberg, L., \& Ritter, P. L. (1997). Parenting styles, adolescents' attributions, and educational outcomes in nine heterogeneous high schools. Child Development, 68(3), 507-529.

http://dx.doi.org/10.1111/j.1467-8624.1997.tb01955.x

González, A. R., Holbein, M. F. D., \& Quilter, S. (2002). High school student's goal orientation and their relationship to perceived parenting styles. Contemporary Educational Psychology, 27(3), 450-470.

http://dx.doi.org/10.1006/ceps.2001.1104

Grolnick, W. S. \& Ryan, R. M. (1989). Parent styles associated with children's selfregulation and competence in school. Journal of Educational Psychology, 81(2), 143-154. http://dx.doi.org/10.1037/0022-0663.81.2.143

Grotevant, H. D. (1998). Adolescent development in family contexts. En W. Damon \& N. Eisenberg (Eds.), Handbook of child psychology (Vol. 3, pp. 1097-1149). New York, New York: Wiley.

Kerr, M. \& Stattin, H. (2000). What parents know, how they know it, and several forms of adolescent adjustment: Further support for a reinterpretation of monitoring. Developmental Psychology, 36(3), 366-380.

http://dx.doi.org/10.1037/0012-1649.36.3.366

Lamborn, S. D., Mounts, N. S., Steinberg, L., \& Dornbush, S. M. (1991). Pattern of competence and adjustment among adolescents from authoritative, authoritarian, indulgent and neglectful families. Child Development, 62(5), 1049-1065.

http://dx.doi.org/10.1111/j.1467-8624.1991.tb01588.x

Lewis, C. C. (1981). The effects of parental firm control: A reinterpretation of findings. Psychological Bulletin, 90(3), 547-563.

http://dx.doi.org/10.1037/0033-2909.90.3.547

Maccoby, E. E. \& Martin, J. A. (1983). Socialization in the context of the family: Parentchild interaction. En E. M. Hetherington \& P. H. Mussen (Eds.), Handbook of child psychology (Vol. 4, pp. 1-101). New York, New York: Wiley. 
Márquez, E. (1995). Hábitos de estudio y personalidad. Distrito Federal, México: Trillas.

Monereo, C. \& Castelló, M. (1997). Las estrategias de aprendizaje. Cómo incorporarlas a la práctica educativa. Barcelona, España: Edebé.

Monereo, C., Castelló, M., Clariana, M., Palma, M., \& Pérez, M. L. (1999). Estrategias de enseñanza y aprendizaje. Formación del profesorado y aplicación en la escuela. Barcelona, España: Editorial Graó.

Nisbet, J. \& Shucksmith, J. (1987). Estrategias de aprendizaje. Madrid, España: Santillana/Aula XXI.

Nyarko, K. (2011). The influence of authoritative parenting style on adolescents' academic achievement. American Journal of Social and Management Sciences, 2(3), 278-282.

http://dx.doi.org/10.5251/ajsms.2011.2.3.278.282

Oliva, A. (2006). Relaciones familiares y desarrollo adolescente. Anuario de Psicología, 37(3), 209-223. Recuperado de

http://www.raco.cat/index.php/AnuarioPsicologia/article/view/61838/82584

Oliva, A. \& Parra, A. (2001). Autonomía emocional durante la adolescencia. Infancia y Aprendizaje, 24(2), 181-196.

http://dx.doi.org/10.1174/021037001316920726

Oliva, A., Parra, A., \& Arranz, E. (2008). Estilos relacionales parentales y ajuste adolescente. Infancia y Aprendizaje, 31(1), 93-106.

http://dx.doi.org/10.1174/021037008783487093

Osorio de Rebellón Yohn, A., Rivas Borrell, S., de Irala Estévez, J., Calatrava, M., \& López del Burgo, C. (2009). Evaluación de los estilos educativos parentales en una muestra de estudiantes filipinos: implicaciones educativas. Revista Panamericana de Pedagogía, 14, 13-37. Recuperado de

http://www.unav.es/icf/main/top/2010/Osorio_Estilos-educativos-parentales.pdf

Pelegrina, S., García Linares, M. C., \& Casanova, P. F. (2002). Los estilos educativos de los padres y la competencia académica de los adolescentes. Infancia y Aprendizaje, 25(2), 147-168.

http://dx.doi.org/10.1174/021037002317417796

Peralbo Uzquiano, M. \& Fernández Amado, M. L. (2003). Estructura familiar y rendimiento escolar en educación secundaria obligatoria. Revista Galego-Portuguesa de Psicoloxía e Educación, 8(7), 309-322. Recuperado de http://dialnet.unirioja.es/servlet/articulo?codigo=1039921

Pérez Sánchez, A. M. \& Castejón Costa, J. L. (1998). Un modelo causal-explicativo sobre la influencia de las variables psicosociales en el rendimiento académico. Bordón. Revista de Pedagogía, 50(2), 171-185. Recuperado de

http://dialnet.unirioja.es/servlet/articulo?codigo=54593

Pintrich, P. R. (1999). The role of motivation in promoting and sustaining self-regulated learning. International Journal of Educational Research, 31(6), 459-470.

http://dx.doi.org/10.1016/S0883-0355(99)00015-4 
Richaud de Minzi, M. C. (2002). Inventario acerca de la percepción que tienen los niños y las niñas de las relaciones con sus padres y madres: versión para 4 a 6 años. Revista Interamericana de Psicología, 36(1-2), 149-165. Recuperado de http://www.redalyc.org/articulo.oa?id=28436210

Richaud de Minzi, M. C. (2005). Versión abreviada del Inventario de la percepción de los hijos acerca de las relaciones con sus padres para adolescentes. Psicodiagnosticar, 15, 99-106.

Schaefer, E. S. (1965). Children's reports of parental behavior: An inventory. Child Development, 36(2), 413-424.

http://www.ncbi.nlm.nih.gov/pubmed/14300862

Sobrado Fernández, L. M., Cauce Santalla, A. I., \& Rial Sánchez, R. (2002). Las habilidades de aprendizaje y estudio en la educación secundaria: estrategias orientadoras de mejora. Tendencias Pedagógicas, 7, 155-177. Recuperado de https://repositorio.uam.es/handle/10486/4833

Steinberg, L., Lamborn, S. D., Darling, N., Mounts, N. S., \& Dornbusch, S. M. (1994). Over-time changes in adjustment and competence among adolescents from authoritative, authoritarian, indulgent, and neglectful families. Child Development, 65(3), 754777. http://dx.doi.org/doi:10.1111/j.1467-8624.1994.tb00781.x

Steinberg, L., Lamborn, S. D., Dornbusch, S. M., \& Darling, N. (1992). Impact of parenting practices on adolescent achievement: Authoritative parenting, school involvement, and encouragement to succeed. Child Development, 63(5), 1266-1281. http://dx.doi.org/10.1111/j.1467-8624.1992.tb01694.x

Stewart, S. M. \& Bond, M. H. (2002). A critical look at parenting research from the mainstream: Problems uncovered while adapting Western research to non-Western cultures. British Journal of Developmental Psychology, 20(3), 379-392. http://dx.doi.org/10.1348/026151002320620389

Strucchi, E. (1991). LASSI. Inventario de estrategias de aprendizaje y estudio. Buenos Aires, Argentina: Psicoteca.

Vallejo Casarín, A. \& Mazadiego Infante, T. J. (2006). Familia y rendimiento académico. Revista de Educación y Desarrollo, 5, 55-59. Recuperado de http://www.cucs.udg.mx/revistas/edu_desarrollo/anteriores/5/005_Vallejo.pdf

Weinstein, C. \& Mayer, R. (1986). The teaching of learning strategies. En M. C. Wittrock (Ed.), Handbook of research on teaching (pp. 315-327). New York, New York: Collier Macmillan Publishing Company.

Weinstein, C. E. \& Palmer, D. R. (1988). LASSI: The learning and study strategies inventory. Clearwater, Florida: H \& H Publishing Company. 